Bull. Fac. Agric., Cairo Univ.,65: 104-118 (2014).

\title{
PATH ANALYSIS AS STATISTICAL MODEL FOR SCREENING NEW LINES OF SNAP BEAN ( Phaseolus vulgaris L.)
}

(Received: 3. 2. 2014)

\author{
By \\ R.M.Galal, W.W.M.Shefei*and S. A. Farag* \\ Vegetable Research Department, Horticulture Research Institute, and *The Central Laboratory for \\ Design and Statistical Analysis Research, Agricultural Research Center, Giza, Egypt
}

\begin{abstract}
Four field experiments were conducted at Sids Horticulture Research Station, Beni-Sueif Governorate, Horticulture Research Institute, Agriculture Research Center, during fall 2011, summer 2012, fall 2012 and summer 2013 seasons on snap bean (Phaseolus vulgaris L.). Sixteen genotypes (ten new lines and six cultivars) were evaluated for their yield and growth characteristics. Plant height, the number of branches/plant, snap pod length, snap pod thickness, snap pod width, snap pod weight, the number of snap pods/plant, the number of dry seeds/pod, 100-dry seeds weight and early and total snap pods yield were studied. Also, the path analysis was studied. The results showed that Lines 20 and 24 produced the highest snap pod yield/feddan in the four seasons. Results of path analysis showed that early yield, pod weight, the number of pods per plant and the number of branches per plant were the most important contributing traits to the total yield. It is concluded that these results are important for designing selection criteria index in our snap bean breeding program.
\end{abstract}

Key words: path coefficient analysis, phaseolus vulgaris L., snap bean, yield, yield components.

\section{INTRODUCTION}

Snap bean (Phaseolus vulgaris L.) is one of Fabaceae family. It is also known as common, snap, kidney, French or haricot beans (Singh 1999). It is one of the most important food crops in Egypt and consumed as a cooked vegetable either as dry seeds or green pods.

Many investigators reported that the vegetative growth and the total and exportable yield as well as pod quality of snap bean are greatly affected by the genotypes (Nassar 1986, El-Sayed 1990, 1996, Mohamed 1997, Dahiya et al. 2000, Mohamed 2004 and Araujo et al.2012).

El-Sayed (1990) found a significant difference in pod length, pod thickness, the number of seeds/pod and the the number of snap pods/plant among the different cultivars. Escribano et al. (1994) found that pod width differed significantly among genotypes. Singh et al. (1994) found that the number of snap pods/plant and pod weight were significantly affected by the tested cultivars.
Zhiwei et al. (1995) found significant differences and variation among the cultivars for pod weight. El-Sayed (1996) found significant differences for plant height among different bean genotypes. Mohamed (1997) reported that the six evaluated snap bean cultivars exhibited similarity in the length of the main stem and found significant differences for the number of the developed primary branches, pod length, pod width and 100seeds weight among the different cultivars. Dilana and Tema cultivars produced the greatest early and total yield. Dahiya et al. (2000) found significant differences for plant height and primary branches/plant among the different bean genotypes. Also, Mohamed (2004) found significant differences for plant height, the number of branches/plant, the the number of seeds/pod, 100 -seeds weight and snap pods/plant among the different bean genotypes. Atilla (2007) found a significant difference in pod length, pod width, pod weight, the number of seeds/pod, 100-seeds 
weight, snap pods/plant, early and total yield among the different cultivars. Sofi et al. (2011) found that plant height, the number of seeds/pod, 100 -seeds weight and the number of snap pods/plant were significantly different among the tested cultivars.

Yield is a complex dependent character and it is contributed by several component characters. Direct selection for seed yield is often not very effective and thus indirect selection for some of the associated component traits may be useful.

Path analysis, a method proposed by Wright (1921), permits the partitioning of the correlation coefficients into direct and indirect effects of various traits on variables whose estimates are obtained by multiple regression equations where the variables are previously standardized. Dewey and $\mathrm{Lu}$ (1959) described the advantage of path analysis. It permits the partitioning of the correlation coefficient into its components that measure the direct effect of a predictor variable upon it's response variable. The second component is to measure the direct effects of a predictor variable on the response variable through other predictor variables. Path coefficient analysis is a statistical technique to evaluate the relation among traits.

In this study, the categorization of most effective traits on snap bean yield production was investigated. The path analysis can contribute knowledge on the changes caused by the environment in the inter-relationships between traits of importance in common bean breeding (Coimbra et al., 1998 and 1999, Kurek et al., 2001).

Gravois and Helmes (1992) reported that path analysis has been applied in crop breeding. Although correlation coefficients among traits are frequently present, they are not incorporated into a path analysis to investigate the relative direct and indirect influence of each trait on yield. According to Board et al. (1997), path coefficient is a standardized partial regression coefficient that has been used to organize and present the casual relationships between predictor and response variables through a path diagram that is based on experimental results. Ribeiro et al. (2003) reported that path analysis showed that the correlation between yield and its components was modified by the genotype by year interaction. Their results suggested that more years of evaluation are necessary to obtain more reliable and useful estimates. Mehra and Singh (2012) reported that the relative importance of the number of primary branches per plant and the number of pods per plant can not be ignored when selection is practiced for improving the pod yield in French bean. For effective selection, greater emphasis should be laid on the number of pods per plant, the number of pods per cluster and the number of primary branches per plant.

The first objective of this study was to evaluate ten new lines and six cultivars of snap bean for growth and green pod yield and its components under Middle Egypt growing conditions. Also, this study aimed to confirm the nature of interrelationship as well as the direct and indirect effects of yield components in the sixteen evaluated genotypes.

\section{MATERIALS AND METHODS}

Four field experiments were conducted at Sids Horticulture Research Station, Beni-Sueif Governorate, Horticulture Research Institute, Agricultural Research Center, Giza, Egypt.

Seeds of sixteen snap bean (Phaseolus vulgaris L.) genotypes (ten new lines and six commercial cultivars) were sown in the growing seasons of fall 2011(September $9^{\text {th }}$ ), summer 2012(March $1^{\text {st }}$ ), fall 2012 (September 10 ${ }^{\text {th }}$ ) and summer 2013(March $5^{\text {st }}$ ). (Table 1). Cultivar names are Tema, Paulista, Xera, Bronco, Giza 3 and Baslem.

The lines were selected by Galal (2004) in his breeding program. Lines 18, 20, 24, 27, 38, 143, and Line 156 were produced by hybridization between Bronco x BARC-RR-3 genotypes, while, Line 5-2, Line 18-2, Line 41-2 were produced by hybridization between Aurora x Olathe. These lines and cultivars were produced in Sids Horticulture Research Station.

In each season, sixteen genotypes were arranged in a randomized complete block design (RCBD) with three replicates. Seeds of each genotype were planted in hills, $5 \mathrm{~cm}$ apart on rows $4 \mathrm{~m}$ in length and $60 \mathrm{~cm}$ in width. Each experimental unit consisted of six rows (three rows 
producd snap pods and three rows produced dry seed yield). The soil of the experiment was clay loam. Also, different agricutltural production practices i.e. irrigation, fertilization and pest management were applied as recomonded by Egyptian Ministry of Agriculture. compared using Duncan's multiple range test Duncan (1955).

Path analysis was made on the basis of phenotypic correlation coefficients taking the total snap pod yield as effect and the remaining estimated characters as cause. Direct and indirect

Table (1): Monthly means of day temperatures during the fall and summer seasons of 2011, 2012 and 2013 at Beni-Suief Governorate, Egypt.

\begin{tabular}{|c|c|c|c|c|}
\hline \multicolumn{5}{|c|}{ Temperature ${ }^{\circ} \mathrm{C}$} \\
\hline & \multicolumn{5}{|c|}{ Fall season 2011 } & \multicolumn{2}{c|}{ Fall season 2012 } \\
\hline Month & Maximum & Minimum & Maximum & Minimum \\
\hline September & 32.2 & 19.3 & 33.8 & 20.3 \\
October & 31.4 & 18.1 & 31.5 & 18.6 \\
November & 24.7 & 11.2 & 24.7 & 12.9 \\
December & 20.4 & 8.5 & 21.3 & 8.7 \\
\hline & Summer season2012 & Summer season2013 \\
\hline March & 29.2 & 13.4 & 28.8 & 13.2 \\
April & 31.1 & 14.8 & 29.7 & 15.3 \\
Mayo & 35.6 & 20.5 & 35.0 & 21.2 \\
June & 37.5 & 23.8 & 37.4 & 24.9 \\
\hline
\end{tabular}

Ten plants chosen at random from each plot after 60 days (beginning of pod formation) from planting were used for recording plant height and the number of branches/plant.

Twenty marketable pods were taken at random from each experimental plot to determine pod length, pod thickness, pod width (the width of pod was measured as the distance from side wall to side wall at the largest section of the pod), and pod weight.

Number of snap pods/plant was recorded on five plants labeled at random from each experimental plot, and their the number of snap pods was counted in each harvest, then the total the number was divided by five.

Thirty dry pods were taken at random from each plot to determine the number of dry seeds/pod and 100- dry seed weight.

Early snap pod yield, in each experimental plot was harvested twice both seasons and their early snap pod yield (Tonlfeddan) was estimated.

Total snap pod yield, in each experimental plot was harvested five times at one week intervals in both seasons and their snap pod weight was used to estimate the total yield as (Tonlfeddan).

\subsection{Statistical analysis}

The statistical analysis was conducted by using the computer program MSTAT-C. Means were effects of component characters on snap pods yield were examined using path coefficient analysis as outlined by Dewey and Lu (1959).

\section{RESULTS AND DISCUSSION}

\subsection{Vegetative growth and yield components}

3.1.1. Plant height

Data presented in Table (2) indicated that Line 18, 20, 38, 156 and Paulista genotypes gave tallest plants in the four seasons followed by Line 27 and Tema genotypes. These results are in agreement with those obtained by El-Sayed (1996), Dahiya et al. (2000), Mohamed (2004) and Sofi et al. (2011) who found significant differences for plant height among different bean genotypes. Whereas, Mohamed (1997) reported that the six evaluated snap bean cultivars exhibited similarity in length of the main stem. Also, Araujo et al. (2012) found no significant difference in plant height of the different cultivars under their experimental conditions.

\subsubsection{The number of branches/plant}

Data presented in Table (2) indicated that Tema, Bronco and Giza 3 genotypes gave high values for the number of branches/plant in the four seasons followed by Lines 18, 20, 24 and 27. Mohamed (1997) reported that the evaluated six genotypes, however, differed in the the number of 
Table (2): Mean values of plant height,number of branches/plant,snap pod length and snap pod thickness of 16 bean genotypes evaluated in fall and summer seasons of 2011,2012 and 2013.

\begin{tabular}{|c|c|c|c|c|c|c|c|c|}
\hline \multirow[t]{2}{*}{ Genotype } & \multicolumn{4}{|c|}{ Plant height $(\mathrm{cm})$} & \multicolumn{4}{|c|}{ Number of branches/plant } \\
\hline & $\begin{array}{c}\text { Fall } \\
2011\end{array}$ & $\begin{array}{c}\text { Summer } \\
2012\end{array}$ & $\begin{array}{c}\text { Fall } \\
2012\end{array}$ & $\begin{array}{c}\text { Summer } \\
2013\end{array}$ & $\begin{array}{c}\text { Fall } \\
2011\end{array}$ & $\begin{array}{c}\text { Summer } \\
2012\end{array}$ & $\begin{array}{c}\text { Fall } \\
2012\end{array}$ & $\begin{array}{c}\text { Summer } \\
2013\end{array}$ \\
\hline Line 18 & $44.7 \mathrm{a}$ & $42.9 \mathrm{a}$ & $42.6 \mathrm{ab}$ & $44.7 \mathrm{ab}$ & $3.2 \mathrm{bcd}$ & $3.8 \mathrm{a}$ & $3.3 \mathrm{abc}$ & $3.7 \mathrm{ab}$ \\
\hline Line 20 & $43.5 \mathrm{ab}$ & $42.2 \mathrm{ab}$ & 40.6 abc & $41.8 \mathrm{abcd}$ & $3.2 \mathrm{abc}$ & $3.3 \mathrm{abcd}$ & $3.0 \mathrm{bc}$ & $3.1 \mathrm{def}$ \\
\hline Line 24 & 41.4 abcde & $39.8 \mathrm{abcd}$ & $38.3 \mathrm{bcd}$ & $39.6 \mathrm{cde}$ & $3.5 \mathrm{ab}$ & $3.0 \mathrm{cde}$ & $3.4 \mathrm{ab}$ & 3.3 bcde \\
\hline Line 27 & $42.9 \mathrm{ab}$ & $41.0 \mathrm{abcd}$ & $41.2 \mathrm{abc}$ & $40.4 \mathrm{~cd}$ & $3.0 \mathrm{bcd}$ & $3.5 \mathrm{abc}$ & $3.3 \mathrm{abc}$ & $3.1 \mathrm{def}$ \\
\hline Line 38 & 41.8 abcd & $41.0 \mathrm{abcd}$ & $40.3 \mathrm{abcd}$ & 41.4 abcd & $3.4 \mathrm{abc}$ & $2.7 \mathrm{e}$ & $2.9 \mathrm{bc}$ & $2.7 \mathrm{f}$ \\
\hline Line 143 & $39.1 \mathrm{cde}$ & 38.7 abcd & $38.7 \mathrm{bcd}$ & $40.6 \mathrm{~cd}$ & $3.3 \mathrm{abc}$ & $3.0 \mathrm{cde}$ & $3.0 \mathrm{bc}$ & $3.1 \mathrm{def}$ \\
\hline Line 156 & $42.5 \mathrm{abc}$ & $41.6 \mathrm{abc}$ & $41.6 \mathrm{abc}$ & $43.6 \mathrm{abc}$ & $3.4 \mathrm{abc}$ & $3.7 \mathrm{ab}$ & $3.0 \mathrm{bc}$ & $3.0 \mathrm{ef}$ \\
\hline Line 5-2 & 40.8 bcde & $38.0 \mathrm{bcd}$ & $43.3 \mathrm{a}$ & $41.8 \mathrm{abcd}$ & $3.0 \mathrm{bcd}$ & 3.2 abcde & $3.0 \mathrm{bc}$ & $3.0 \mathrm{ef}$ \\
\hline Line 18-2 & 40.4 bcde & $36.9 \mathrm{~cd}$ & $41.8 \mathrm{abc}$ & $40.6 \mathrm{~cd}$ & $2.6 \mathrm{~d}$ & $2.7 \mathrm{e}$ & $2.7 \mathrm{c}$ & $3.0 \mathrm{ef}$ \\
\hline Line 41-2 & $38.1 \mathrm{e}$ & $39.0 \mathrm{abcd}$ & $37.7 \mathrm{~cd}$ & $38.6 \mathrm{de}$ & $3.0 \mathrm{bcd}$ & $2.9 \mathrm{cde}$ & $3.0 \mathrm{bc}$ & $2.9 \mathrm{ef}$ \\
\hline Tema & 40.35 bcde & $41.5 \mathrm{abc}$ & $42.6 \mathrm{ab}$ & $44.8 \mathrm{a}$ & $3.8 \mathrm{a}$ & $3.8 \mathrm{a}$ & $3.9 \mathrm{a}$ & $3.5 \mathrm{abcd}$ \\
\hline Paulista & 41.50 abcde & 40.8 abcd & $42.7 \mathrm{ab}$ & 42.6 abcd & $3.2 \mathrm{bcd}$ & 3.1 bcde & $3.8 \mathrm{a}$ & 3.3 bcde \\
\hline Xera & $38.90 \mathrm{cde}$ & 39.4 abcd & $39.5 \mathrm{abcd}$ & $40.8 \mathrm{bcd}$ & $2.9 \mathrm{~cd}$ & $2.7 \mathrm{de}$ & $2.8 \mathrm{c}$ & 3.2 cde \\
\hline Bronco & $38.23 \mathrm{de}$ & 38.7 abcd & $39.5 \mathrm{abcd}$ & $40.0 \mathrm{~cd}$ & $3.6 \mathrm{ab}$ & $3.5 \mathrm{abc}$ & $3.6 \mathrm{a}$ & $3.6 \mathrm{abc}$ \\
\hline Giza3 & 40.23 bcde & 40.7 abcd & $42.6 \mathrm{ab}$ & $40.5 \mathrm{~cd}$ & $3.2 \mathrm{abc}$ & $3.5 \mathrm{abc}$ & $3.3 \mathrm{abc}$ & $3.8 \mathrm{a}$ \\
\hline Baslim & $34.50 \mathrm{f}$ & $36.6 \mathrm{~d}$ & $35.9 \mathrm{~d}$ & $36.0 \mathrm{e}$ & $2.9 \mathrm{bcd}$ & 3.2 bcde & $3.0 \mathrm{bc}$ & $3.0 \mathrm{ef}$ \\
\hline \multirow[t]{2}{*}{ Genotype } & \multicolumn{4}{|c|}{ Snap pod length ( cm ) } & \multicolumn{4}{|c|}{ Snap pod thickness ( mm ) } \\
\hline & $\begin{array}{c}\text { Fall } \\
2011\end{array}$ & $\begin{array}{c}\text { Summer } \\
2012\end{array}$ & $\begin{array}{c}\text { Fall } \\
2012\end{array}$ & $\begin{array}{c}\text { Summer } \\
2013\end{array}$ & $\begin{array}{c}\text { Fall } \\
2011\end{array}$ & $\begin{array}{c}\text { Summer } \\
2012\end{array}$ & $\begin{array}{c}\text { Fall } \\
2012\end{array}$ & $\begin{array}{c}\text { Summer } \\
2013\end{array}$ \\
\hline Line 18 & $13.2 \mathrm{a}$ & $11.6 \mathrm{a}$ & $13.5 \mathrm{a}$ & $12.0 \mathrm{ab}$ & 7.1 bcde & $6.6 \mathrm{ab}$ & $6.9 \mathrm{~cd}$ & $6.7 \mathrm{cde}$ \\
\hline Line 20 & $13.1 \mathrm{a}$ & $11.6 \mathrm{a}$ & $13.0 \mathrm{a}$ & $11.8 \mathrm{abc}$ & $6.4 \mathrm{efg}$ & $6.7 \mathrm{ab}$ & $5.7 \mathrm{f}$ & $6.8 \mathrm{cde}$ \\
\hline Line 24 & $11.3 \mathrm{c}$ & $10.7 \mathrm{ab}$ & $11.8 \mathrm{c}$ & $10.8 \mathrm{cdef}$ & $6.8 \mathrm{def}$ & $6.8 \mathrm{ab}$ & $6.8 \mathrm{cde}$ & $7.0 \mathrm{cde}$ \\
\hline Line 27 & $12.0 \mathrm{~b}$ & 9.6 abc & $12.2 \mathrm{~b}$ & $9.8 \mathrm{fg}$ & $7.2 \mathrm{bcd}$ & $7.2 \mathrm{ab}$ & $7.4 \mathrm{bc}$ & $7.0 \mathrm{cde}$ \\
\hline Line 38 & $12.4 \mathrm{~b}$ & 9.9 abc & $13.0 \mathrm{a}$ & $10.0 \mathrm{efg}$ & $7.8 \mathrm{~b}$ & $7.7 \mathrm{ab}$ & $7.4 \mathrm{bc}$ & $7.7 \mathrm{bc}$ \\
\hline Line 143 & $13.2 \mathrm{a}$ & $10.5 \mathrm{ab}$ & $13.0 \mathrm{a}$ & $10.8 \mathrm{cdef}$ & $6.9 \mathrm{cdef}$ & $7.1 \mathrm{ab}$ & $7.0 \mathrm{bcd}$ & $7.2 \mathrm{~cd}$ \\
\hline Line 156 & $12.5 \mathrm{~b}$ & $10.2 \mathrm{ab}$ & $12.8 \mathrm{ab}$ & $10.3 \mathrm{defg}$ & $7.4 \mathrm{bcd}$ & $7.3 \mathrm{ab}$ & $6.8 \mathrm{cde}$ & $7.3 \mathrm{~cd}$ \\
\hline Line 5-2 & $8.8 \mathrm{f}$ & 8.9 bc & $9.2 \mathrm{f}$ & $8.8 \mathrm{hi}$ & $8.9 \mathrm{a}$ & $7.2 \mathrm{ab}$ & $8.8 \mathrm{a}$ & $9.3 \mathrm{a}$ \\
\hline Line 18-2 & $10.1 \mathrm{e}$ & $5.9 \mathrm{~d}$ & $10.5 \mathrm{de}$ & $9.6 \mathrm{gh}$ & $8.8 \mathrm{a}$ & $8.8 \mathrm{a}$ & $8.9 \mathrm{a}$ & $8.6 \mathrm{ab}$ \\
\hline Line 41-2 & $10.4 \mathrm{de}$ & $7.7 \mathrm{~cd}$ & $11.0 \mathrm{~d}$ & $8.0 \mathrm{i}$ & $8.8 \mathrm{a}$ & $8.5 \mathrm{a}$ & $8.8 \mathrm{a}$ & $8.9 \mathrm{a}$ \\
\hline Tema & $13.3 \mathrm{a}$ & $11.8 \mathrm{a}$ & $13.0 \mathrm{a}$ & $12.5 \mathrm{a}$ & $6.1 \mathrm{fg}$ & $6.0 \mathrm{~b}$ & $6.3 \mathrm{def}$ & $6.3 \mathrm{de}$ \\
\hline Paulista & $10.7 \mathrm{~d}$ & $11.0 \mathrm{ab}$ & $11.0 \mathrm{~cd}$ & $11.8 \mathrm{abc}$ & $6.1 \mathrm{fg}$ & $6.1 \mathrm{~b}$ & $6.0 \mathrm{ef}$ & $6.0 \mathrm{e}$ \\
\hline Xera & $10.4 \mathrm{de}$ & $10.9 \mathrm{ab}$ & $11.2 \mathrm{c}$ & $11.0 \mathrm{cde}$ & $5.7 \mathrm{~g}$ & $5.9 \mathrm{~b}$ & $5.9 \mathrm{f}$ & $5.9 \mathrm{e}$ \\
\hline Bronco & $10.2 \mathrm{de}$ & $10.9 \mathrm{ab}$ & $10.5 \mathrm{de}$ & $11.0 \mathrm{cde}$ & $7.2 \mathrm{bcd}$ & $7.2 \mathrm{ab}$ & $7.8 \mathrm{~b}$ & $6.7 \mathrm{cde}$ \\
\hline Giza3 & $12.2 \mathrm{~b}$ & $11.2 \mathrm{ab}$ & $10.5 \mathrm{de}$ & $11.1 \mathrm{bcd}$ & 6.9 cdef & $6.8 \mathrm{ab}$ & $7.0 \mathrm{bcd}$ & $6.7 \mathrm{cde}$ \\
\hline Baslim & $10.5 \mathrm{de}$ & $10.6 \mathrm{ab}$ & $10.8 \mathrm{de}$ & 10.8 cdef & $7.7 \mathrm{bc}$ & $7.2 \mathrm{ab}$ & $7.0 \mathrm{bcd}$ & $6.9 \mathrm{cde}$ \\
\hline
\end{tabular}

Means followed by the same letters within each column do not differ significantly according to Duncan's Multiple Range test at the $5 \%$ level. 
the developed primary branches. The cv. Giza 3 distinctively had the least the number of primary branches (4.1) while, cv. Bronco had (4.6) and cv. Tema had (4.9) branches/plant. Dahiya et al. (2000) found significant differences for primary branches/plant. In snap bean, Mohamed (2004) found that the number of branches/plant was significantly different among the ten genotypes.

\subsubsection{Snap pod length}

Data presented in Table (2) indicated that Lines 18, 20 and Tema genotypes produced the tallest pods in the four seasons followed by Line 143. ElSayed (1990), Mohamed (1997), Atilla (2007) and Araujo et al. (2012) found a significant difference in pod length among the different cultivars.

\subsubsection{Snap pod thickness}

Data presented in Table 2 indicated that Line 20, Paulista, Tema and Xera genotypes produced the thinnest pods in the four seasons followed by Lines 18, 24 and Line 27. El-Sayed (1990) and Mohamed (1997) found significant differences in pod thickness due to cultivar differences.

\subsubsection{Snap pod width}

Data presented in Table (3) indicated that Lines 27, 5-2, and Line 18-2 produced the widthest pods in the four seasons followed by Baslim genotypes. Escribano et al. (1994) and Atilla (2007) found that pod width differed significantly among genotypes.

\subsubsection{Snap pod weight}

Data presented in Table (3) indicated that Line 20 and Line 24 produced the highest weight of pods in the four seasons. Singh et al. (1994), Zhiwei et al. (1995), Atilla (2007) and Araujo et al. (2012) found significant differences and variation among the cultivars for pod weight.

\subsubsection{The number of dry seeds/pod}

Data presented in Table (3) indicated that Lines $20,143,24,27,18-2$ and Line 5-2 produced the highest number of seeds/pod in the four seasons followed by Lines 18, 38, and Line 18-2, Tema, Paulista, Bronco, Xera and Giza 3 genotypes. ElSayed (1990), Mohamed (2004), Atilla (2007), Sofi et al. (2011) and Araujo et al. (2012) found that the the number of seeds/pod was significantly different among the tested cultivars.

\subsubsection{0- dry seed weight}

Data presented in Table (3) indicated that Giza $3 \mathrm{cv}$. produced the highest 100- dry seed weight in the four seasons followed by Bronco, Tema, Lines 27, 38 and Line 156. On the other side, Lines 18, 20, 18-2, and Line 18-2, Paulista, Xera and Balsim genotypes produced the lightest 100- dry seeds weight in all seasons. Mohamed (1997), Mohamed (2004), Atilla (2007) and Sofi et al. (2011) found that 100-seed weight differed significantly among the studied genotypes. Whereas, Araujo et al. (2012) found that the 100-seed weight was significantly affected by the tested cultivars.

\subsubsection{Number of snap pods/plant}

Data presented in Table (4) indicated that Line 20 produced the highest the number of snap pods/plant in the four seasons followed by Line 24, Tema and Paulista genotypes. . El-Sayed (1990), Singh et al. (1994), Mohamed (2004), Atilla (2007), Sofi et al. (2011) and Araujo et al. (2012) found that the the number of snap pods/plant was significantly affected by the tested cultivars.

\subsubsection{Early snap pod yield}

Data presented in Table (4) for early yield indicated that Line 20 produced the highest value for all seasons followed by Line 24, Tema and Paulista genotypes.

\subsubsection{Total snap pod yield}

Data presented in Table (5) for this character indicated that Line 20 and Line 24 produced the highest yield/feddan in the four seasons followed by Paulista and Tema genotypes. Mohamed (1997) found that Dilana and Tema cultivars produced the greatest early and total yield. Atilla (2007) and Araujo et al. (2012) found that the early and total yield were significantly affected by the tested cultivars.

Accordingly, from the foregoing results, it could be concluded that Line 20 and Line 24 gave the highest values in the fall and summer seasons for both early and total snap yield per feddan.

\subsection{Direct and indirect effects of component characters on snap pod yield}

Path coefficient analysis of the results of the first season between all possible combinations was estimated (Table 6).The analysis appeared to provide a clue to the contribution of various components of the yield to over all pod yields in the genotypes under study.

In the present investigation, the resultant variable was pod yield while the remaining 108 
Table (3): Mean values of snap pod width,snap pod weight, number of dry seeds/pod and 100- dry seeds weight of 16 bean genotypes evaluated in fall and summer seasons of 2011, 2012 and 2013.

\begin{tabular}{|c|c|c|c|c|c|c|c|c|}
\hline \multirow[b]{2}{*}{ Genotype } & \multicolumn{4}{|c|}{ Snap pod width ( mm ) } & \multicolumn{4}{|c|}{ Snap pod weight ( $g$ ) } \\
\hline & $\begin{array}{l}\text { Fall } \\
2011\end{array}$ & $\begin{array}{c}\text { Summer } \\
2012\end{array}$ & Fall 2012 & $\begin{array}{c}\text { Summer } \\
2013\end{array}$ & $\begin{array}{l}\text { Fall } \\
2011\end{array}$ & $\begin{array}{c}\text { Summer } \\
2012\end{array}$ & $\begin{array}{c}\text { Fall } \\
2012\end{array}$ & $\begin{array}{c}\text { Summer } \\
2013\end{array}$ \\
\hline Line 18 & 5.2 abcd & $5.0 \mathrm{abc}$ & $5.3 \mathrm{abcd}$ & $5.2 \mathrm{ab}$ & $3.5 \mathrm{bc}$ & $3.4 \mathrm{bcd}$ & 3.3 bcdef & $3.6 \mathrm{~b}$ \\
\hline Line 20 & 5.0 abcd & $4.8 \mathrm{bc}$ & $5.1 \mathrm{bcd}$ & $5.0 \mathrm{abc}$ & $4.3 \mathrm{a}$ & $4.6 \mathrm{a}$ & $4.6 \mathrm{a}$ & $4.5 \mathrm{a}$ \\
\hline Line 24 & 4.9abcde & 4.9 abc & 5.0 cde & $5.2 \mathrm{ab}$ & $4.7 \mathrm{a}$ & $4.8 \mathrm{a}$ & $4.5 \mathrm{a}$ & $4.7 \mathrm{a}$ \\
\hline Line 27 & 4.4 cde & $4.2 \mathrm{de}$ & $4.5 \mathrm{efg}$ & $4.2 \mathrm{de}$ & $3.1 \mathrm{bcd}$ & 3.3 bcde & $3.1 \mathrm{def}$ & 3.0 ef \\
\hline Line 38 & $5.6 \mathrm{ab}$ & $5.3 \mathrm{ab}$ & $5.7 \mathrm{ab}$ & $5.5 \mathrm{a}$ & $3.5 \mathrm{~b}$ & $3.7 \mathrm{bc}$ & $3.6 \mathrm{bcd}$ & 3.4 bcde \\
\hline Line 143 & $5.5 \mathrm{abc}$ & $5.5 \mathrm{a}$ & $5.6 \mathrm{abc}$ & $5.5 \mathrm{a}$ & $3.4 \mathrm{bc}$ & $3.7 \mathrm{~b}$ & $3.7 \mathrm{bc}$ & $3.5 \mathrm{bcd}$ \\
\hline Line 156 & $5.3 \mathrm{abcd}$ & $5.2 \mathrm{ab}$ & $5.2 \mathrm{bcd}$ & $5.4 \mathrm{ab}$ & $3.5 \mathrm{bc}$ & $3.1 \mathrm{cdef}$ & 3.2 bcdef & $3.1 \mathrm{cdef}$ \\
\hline Line 5-2 & $4.0 \mathrm{e}$ & $3.8 \mathrm{e}$ & $4.0 \mathrm{~g}$ & $4.0 \mathrm{de}$ & $3.2 \mathrm{bc}$ & $3.7 \mathrm{bc}$ & 3.3 bcde & 3.4 bcde \\
\hline Line 18-2 & $4.0 \mathrm{e}$ & $4.0 \mathrm{de}$ & $4.4 \mathrm{fg}$ & $3.8 \mathrm{e}$ & $2.2 \mathrm{e}$ & $2.7 \mathrm{ef}$ & $2.9 \mathrm{ef}$ & $2.4 \mathrm{~g}$ \\
\hline Line 41-2 & 4.7 bcde & $4.5 \mathrm{~cd}$ & $4.8 \mathrm{def}$ & $4.8 \mathrm{bc}$ & $3.1 \mathrm{bcd}$ & $2.9 \mathrm{def}$ & 2.9 ef & $3.1 \mathrm{def}$ \\
\hline Tema & $5.2 \mathrm{abcd}$ & $5.0 \mathrm{abc}$ & 5.0 cde & $5.2 \mathrm{ab}$ & $3.4 \mathrm{~b}$ & $3.8 \mathrm{~b}$ & $3.7 \mathrm{~b}$ & $3.5 \mathrm{bc}$ \\
\hline Paulista & $5.3 \mathrm{abcd}$ & $5.1 \mathrm{abc}$ & $5.2 \mathrm{bcd}$ & $5.2 \mathrm{ab}$ & $3.0 \mathrm{bcd}$ & 3.3 bcdef & 3.2 bcdef & 3.0 def \\
\hline Xera & $5.1 \mathrm{abcd}$ & $5.0 \mathrm{abc}$ & $5.0 \mathrm{cde}$ & $5.0 \mathrm{abc}$ & $2.9 \mathrm{~cd}$ & $2.7 \mathrm{f}$ & $2.7 \mathrm{f}$ & $2.8 \mathrm{fg}$ \\
\hline Bronco & $5.5 \mathrm{ab}$ & $4.8 \mathrm{bc}$ & $5.6 \mathrm{abc}$ & $5.4 \mathrm{ab}$ & $3.4 \mathrm{bc}$ & 3.3 bcde & $3.1 \mathrm{cdef}$ & $3.1 \mathrm{def}$ \\
\hline Giza3 & $5.9 \mathrm{a}$ & $5.5 \mathrm{a}$ & $5.8 \mathrm{a}$ & $5.3 \mathrm{ab}$ & $3.3 \mathrm{bc}$ & $3.3 \mathrm{bcde}$ & $3.5 \mathrm{bcde}$ & 3.3 bcdef \\
\hline Baslim & $4.3 \mathrm{de}$ & $4.2 \mathrm{de}$ & $4.2 \mathrm{~g}$ & $4.5 \mathrm{~cd}$ & $2.6 \mathrm{de}$ & $2.7 \mathrm{ef}$ & $2.7 \mathrm{f}$ & $3.1 \mathrm{def}$ \\
\hline \multirow[b]{2}{*}{ Genotype } & \multicolumn{4}{|c|}{ Number of dry seeds/ pod } & \multicolumn{4}{|c|}{ 100- dry seeds weight ( g ) } \\
\hline & \begin{tabular}{c|} 
Fall \\
2011
\end{tabular} & $\begin{array}{c}\text { Summer } \\
2012\end{array}$ & \begin{tabular}{|l|} 
Fall \\
2012 \\
\end{tabular} & $\begin{array}{c}\text { Summer } \\
2013\end{array}$ & $\begin{array}{l}\text { Fall } \\
2011\end{array}$ & $\begin{array}{c}\text { Summer } \\
2012\end{array}$ & $\begin{array}{c}\text { Fall } \\
2012\end{array}$ & $\begin{array}{c}\text { Summer } \\
2013\end{array}$ \\
\hline Line 18 & $6.0 \mathrm{abc}$ & $5.9 \mathrm{ab}$ & $5.8 \mathrm{ab}$ & $5.3 \mathrm{bcd}$ & $18.4 \mathrm{efg}$ & $17.9 \mathrm{f}$ & $17.2 \mathrm{fg}$ & $17.2 \mathrm{gh}$ \\
\hline Line 20 & $6.4 \mathrm{a}$ & $6.1 \mathrm{a}$ & $6.5 \mathrm{a}$ & $6.0 \mathrm{a}$ & $18.3 \mathrm{efg}$ & 19.7 cdef & $19.5 \mathrm{cdef}$ & $17.7 \mathrm{~g}$ \\
\hline Line 24 & $6.3 \mathrm{ab}$ & $5.3 \mathrm{ab}$ & $5.8 \mathrm{ab}$ & $5.4 \mathrm{abcd}$ & $20.7 \mathrm{de}$ & $22.0 \mathrm{c}$ & $18.3 \mathrm{ef}$ & $20.2 \mathrm{ef}$ \\
\hline Line 27 & $5.9 \mathrm{abc}$ & $5.3 \mathrm{ab}$ & $5.7 \mathrm{ab}$ & $5.6 \mathrm{abcd}$ & $24.7 \mathrm{~b}$ & $25.5 \mathrm{~b}$ & $25.0 \mathrm{ab}$ & $24.5 \mathrm{c}$ \\
\hline Line 38 & $5.7 \mathrm{bcd}$ & $5.9 \mathrm{ab}$ & $5.8 \mathrm{ab}$ & $5.5 \mathrm{abcd}$ & $24.4 \mathrm{bc}$ & $24.5 \mathrm{~b}$ & $23.6 \mathrm{abc}$ & $23.5 \mathrm{~cd}$ \\
\hline Line 143 & $6.5 \mathrm{a}$ & $5.9 \mathrm{a}$ & $6.1 \mathrm{a}$ & $6.0 \mathrm{a}$ & $22.1 \mathrm{~cd}$ & 20.9 cde & 22.1 bcde & $20.3 \mathrm{ef}$ \\
\hline Line 156 & $5.2 \mathrm{de}$ & $5.3 \mathrm{ab}$ & $5.1 \mathrm{ab}$ & $5.0 \mathrm{~d}$ & $24.5 \mathrm{bc}$ & $21.6 \mathrm{~cd}$ & $23.0 \mathrm{abcd}$ & $21.9 \mathrm{de}$ \\
\hline Line 5-2 & $6.3 \mathrm{ab}$ & $5.6 \mathrm{ab}$ & $6.1 \mathrm{a}$ & 5.6 abcd & $18.5 \mathrm{ef}$ & $18.9 \mathrm{ef}$ & 19.9 cdef & $18.3 \mathrm{fg}$ \\
\hline Line 18-2 & $6.1 \mathrm{abc}$ & $5.5 \mathrm{ab}$ & $6.0 \mathrm{ab}$ & $5.3 \mathrm{bcd}$ & $17.6 \mathrm{fg}$ & $17.4 \mathrm{fgh}$ & $16.1 \mathrm{fg}$ & $17.2 \mathrm{gh}$ \\
\hline Line 41-2 & $6.5 \mathrm{a}$ & $5.9 \mathrm{a}$ & $6.3 \mathrm{a}$ & $5.7 \mathrm{abc}$ & $17.3 \mathrm{fg}$ & $14.9 \mathrm{~h}$ & $16.7 \mathrm{fg}$ & $15.4 \mathrm{~h}$ \\
\hline Tema & $5.5 \mathrm{cde}$ & $5.4 \mathrm{ab}$ & $5.8 \mathrm{ab}$ & $5.4 \mathrm{abcd}$ & $26.2 \mathrm{~b}$ & $25.6 \mathrm{~b}$ & $26.5 \mathrm{a}$ & $27.1 \mathrm{~b}$ \\
\hline Paulista & 5.6 bcde & $5.4 \mathrm{ab}$ & $5.4 \mathrm{ab}$ & $5.4 \mathrm{abcd}$ & $18.2 \mathrm{efg}$ & $19.3 \mathrm{def}$ & $18.2 \mathrm{ef}$ & $17.5 \mathrm{gh}$ \\
\hline Xera & $6.2 \mathrm{ab}$ & $5.9 \mathrm{ab}$ & $4.2 \mathrm{~b}$ & $5.8 \mathrm{ab}$ & $18.5 \mathrm{efg}$ & $17.8 \mathrm{fg}$ & $19.2 \mathrm{def}$ & $16.9 \mathrm{gh}$ \\
\hline Bronco & 5.4 cde & $5.6 \mathrm{ab}$ & $5.7 \mathrm{ab}$ & $5.4 \mathrm{abcd}$ & $25.5 \mathrm{~b}$ & $25.6 \mathrm{~b}$ & $27.0 \mathrm{a}$ & $27.1 \mathrm{~b}$ \\
\hline Giza3 & $5.9 \mathrm{abcd}$ & $5.3 \mathrm{ab}$ & $5.8 \mathrm{ab}$ & $5.3 \mathrm{bcd}$ & $28.8 \mathrm{a}$ & $28.0 \mathrm{a}$ & $26.7 \mathrm{a}$ & $30.1 \mathrm{a}$ \\
\hline Baslim & $5.0 \mathrm{e}$ & $5.05 \mathrm{~b}$ & $5.1 \mathrm{ab}$ & $5.1 \mathrm{~cd}$ & $15.9 \mathrm{~g}$ & $15.3 \mathrm{gh}$ & $13.3 \mathrm{~g}$ & $16.6 \mathrm{gh}$ \\
\hline
\end{tabular}

Means followed by the same letters within each column do not differ significantly according to Duncan's Multiple Range test at the $5 \%$ level. 
Table (4): Mean values of the number of snap pods/plant and early snap podsyield of 16 bean genotypes evaluated in the fall and summer seasons of 2011, 2012 and 2013.

\begin{tabular}{|c|c|c|c|c|c|c|c|c|}
\hline \multirow{2}{*}{ Genotype } & \multicolumn{4}{|c|}{ Number of snap pods/plant } & \multicolumn{3}{c|}{ Early snap pods yield (Ton/feddan ) } \\
\cline { 2 - 10 } & $\begin{array}{c}\text { Fall } \\
\mathbf{2 0 1 1}\end{array}$ & Summer 2012 & $\begin{array}{c}\text { Fall } \\
\mathbf{2 0 1 2}\end{array}$ & Summer 2013 & $\begin{array}{c}\text { Fall } \\
\mathbf{2 0 1 1}\end{array}$ & Summer 2012 & $\begin{array}{c}\text { Fall } \\
\mathbf{2 0 1 2}\end{array}$ & $\begin{array}{c}\text { Summer } \\
\mathbf{2 0 1 3}\end{array}$ \\
\hline Line 18 & $41.3 \mathrm{cde}$ & $41.7 \mathrm{bcd}$ & $42.5 \mathrm{bc}$ & $40.8 \mathrm{~cd}$ & $1.77 \mathrm{~cd}$ & $1.97 \mathrm{cde}$ & $1.90 \mathrm{de}$ & $2.07 \mathrm{c}$ \\
\hline Line 20 & $49.6 \mathrm{ab}$ & $49.4 \mathrm{a}$ & $51.1 \mathrm{a}$ & $52.9 \mathrm{a}$ & $2.95 \mathrm{a}$ & $3.33 \mathrm{a}$ & $3.25 \mathrm{a}$ & $3.12 \mathrm{a}$ \\
\hline Line 24 & $50.3 \mathrm{a}$ & $50.1 \mathrm{a}$ & $49.3 \mathrm{a}$ & $48.3 \mathrm{~b}$ & $2.85 \mathrm{a}$ & $3.03 \mathrm{ab}$ & $2.88 \mathrm{bc}$ & $2.78 \mathrm{~b}$ \\
\hline Line 27 & $44.2 \mathrm{bcd}$ & $45.8 \mathrm{ab}$ & $42.4 \mathrm{bc}$ & $41.7 \mathrm{c}$ & $2.08 \mathrm{bc}$ & $1.85 \mathrm{de}$ & $1.92 \mathrm{de}$ & $1.85 \mathrm{~cd}$ \\
\hline Line 38 & $40.4 \mathrm{cde}$ & $39.5 \mathrm{cde}$ & $39.4 \mathrm{~cd}$ & $37.8 \mathrm{cdef}$ & $1.52 \mathrm{de}$ & $1.73 \mathrm{e}$ & $1.63 \mathrm{e}$ & $1.62 \mathrm{~d}$ \\
\hline Line 143 & $40.2 \mathrm{cde}$ & $35.2 \mathrm{f}$ & $39.3 \mathrm{~cd}$ & $35.5 \mathrm{ef}$ & $1.33 \mathrm{efg}$ & $1.20 \mathrm{f}$ & $1.17 \mathrm{f}$ & $1.15 \mathrm{e}$ \\
\hline Line 156 & $34.2 \mathrm{f}$ & $38.4 \mathrm{def}$ & $37.6 \mathrm{~cd}$ & $35.9 \mathrm{ef}$ & $2.03 \mathrm{bc}$ & $2.13 \mathrm{cde}$ & $1.93 \mathrm{de}$ & $2.00 \mathrm{c}$ \\
\hline Line 5-2 & $38.1 \mathrm{ef}$ & $41.3 \mathrm{bcd}$ & $36.2 \mathrm{~cd}$ & $37.6 \mathrm{cdef}$ & $1.03 \mathrm{~g}$ & $1.23 \mathrm{f}$ & $1.03 \mathrm{f}$ & $1.00 \mathrm{e}$ \\
\hline Line 18-2 & $38.6 \mathrm{def}$ & $36.2 \mathrm{ef}$ & $34.7 \mathrm{~d}$ & $34.7 \mathrm{f}$ & $1.07 \mathrm{fg}$ & $1.20 \mathrm{f}$ & $1.00 \mathrm{f}$ & $1.03 \mathrm{e}$ \\
\hline Line 41-2 & $40.3 \mathrm{cde}$ & $39.4 \mathrm{cdef}$ & $38.9 \mathrm{~cd}$ & $39.8 \mathrm{cde}$ & $1.83 \mathrm{c}$ & $1.82 \mathrm{e}$ & $1.63 \mathrm{e}$ & $1.77 \mathrm{~cd}$ \\
\hline Tema & $48.6 \mathrm{ab}$ & $47.8 \mathrm{a}$ & $49.7 \mathrm{a}$ & $48.4 \mathrm{~b}$ & $2.65 \mathrm{a}$ & $2.92 \mathrm{~b}$ & $2.98 \mathrm{ab}$ & $2.70 \mathrm{~b}$ \\
\hline Paulista & $45.2 \mathrm{abc}$ & $43.4 \mathrm{bc}$ & $48.4 \mathrm{a}$ & $45.9 \mathrm{~b}$ & $2.87 \mathrm{a}$ & $2.73 \mathrm{~b}$ & $2.57 \mathrm{c}$ & $2.68 \mathrm{~b}$ \\
\hline Xera & $40.5 \mathrm{cde}$ & $41.3 \mathrm{bcd}$ & $38.7 \mathrm{~cd}$ & $39.2 \mathrm{cde}$ & $1.82 \mathrm{~cd}$ & $1.98 \mathrm{cde}$ & $1.95 \mathrm{de}$ & $1.90 \mathrm{~cd}$ \\
\hline Bronco & $41.9 \mathrm{cde}$ & $40.6 \mathrm{cde}$ & $39.6 \mathrm{~cd}$ & $41.1 \mathrm{c}$ & $1.97 \mathrm{bc}$ & $2.23 \mathrm{~cd}$ & $2.18 \mathrm{~d}$ & $2.07 \mathrm{c}$ \\
\hline Giza3 & $44.9 \mathrm{abc}$ & $43.2 \mathrm{bc}$ & $47.0 \mathrm{ab}$ & $46.2 \mathrm{~b}$ & $2.20 \mathrm{~b}$ & $2.27 \mathrm{c}$ & $1.95 \mathrm{de}$ & $1.93 \mathrm{c}$ \\
\hline Baslim & $37.2 \mathrm{ef}$ & $37.8 \mathrm{def}$ & $37.9 \mathrm{~cd}$ & $36.7 \mathrm{def}$ & $1.35 \mathrm{ef}$ & $1.30 \mathrm{f}$ & $1.13 \mathrm{f}$ & $1.07 \mathrm{e}$ \\
\hline
\end{tabular}

Means followed by the same letters within each column do not differ significantly according to Duncan's Multiple Range test at the $5 \%$ level.

Table (5): Mean values of the total snap pod yield of 16 bean genotypes evaluated in the fall and summer seasons of 2011, 2012 and 2013.

\begin{tabular}{|c|c|c|c|c|}
\hline \multirow{2}{*}{ Genotype } & \multicolumn{3}{|c|}{ Total snap pods yield ( Ton/feddan) } \\
\cline { 2 - 5 } & Fall 2011 & $\begin{array}{c}\text { Summer } \\
\mathbf{2 0 1 2}\end{array}$ & Fall 2012 & $\begin{array}{c}\text { Summer } \\
\mathbf{2 0 1 3}\end{array}$ \\
\hline Line 18 & $5.50 \mathrm{~b}$ & $5.23 \mathrm{bc}$ & $5.23 \mathrm{bcd}$ & $5.03 \mathrm{bc}$ \\
\hline Line 20 & $6.37 \mathrm{a}$ & $6.46 \mathrm{a}$ & $6.20 \mathrm{a}$ & $6.43 \mathrm{a}$ \\
\hline Line 24 & $6.23 \mathrm{a}$ & $6.08 \mathrm{a}$ & $6.17 \mathrm{a}$ & $4.67 \mathrm{bcde}$ \\
\hline Line 27 & $5.00 \mathrm{c}$ & $5.02 \mathrm{bc}$ & $4.67 \mathrm{defg}$ & $4.32 \mathrm{defg}$ \\
\hline Line 38 & $4.63 \mathrm{def}$ & $4.90 \mathrm{bcd}$ & $4.60 \mathrm{efg}$ & $4.10 \mathrm{efgh}$ \\
\hline Line 143 & $4.50 \mathrm{efg}$ & $4.03 \mathrm{e}$ & $4.42 \mathrm{fgh}$ & $5.10 \mathrm{~b}$ \\
\hline Line 156 & $4.85 \mathrm{cde}$ & $4.87 \mathrm{bcd}$ & $5.28 \mathrm{~b}$ & $3.75 \mathrm{gh}$ \\
\hline Line 5-2 & $4.17 \mathrm{gh}$ & $3.98 \mathrm{e}$ & $4.10 \mathrm{gh}$ & $3.97 \mathrm{fg}$ \\
\hline Line 18-2 & $4.07 \mathrm{~h}$ & $4.07 \mathrm{e}$ & $4.10 \mathrm{gh}$ & $4.13 \mathrm{efgh}$ \\
\hline Line 41-2 & $4.42 \mathrm{fgh}$ & $4.40 \mathrm{de}$ & $4.30 \mathrm{fgh}$ & $4.90 \mathrm{bcd}$ \\
\hline Tema & $5.47 \mathrm{~b}$ & $5.37 \mathrm{~b}$ & $5.27 \mathrm{bc}$ & $5.13 \mathrm{~b}$ \\
\hline Paulista & $5.25 \mathrm{bc}$ & $5.40 \mathrm{~b}$ & $5.17 \mathrm{bcde}$ & $4.43 \mathrm{cdef}$ \\
\hline Xera & $4.83 \mathrm{cdef}$ & $4.78 \mathrm{~cd}$ & $4.65 \mathrm{efg}$ & $4.93 \mathrm{bcd}$ \\
\hline Bronco & $4.77 \mathrm{def}$ & $5.10 \mathrm{bc}$ & $5.00 \mathrm{bcde}$ & $4.45 \mathrm{cdef}$ \\
\hline Giza3 & $5.00 \mathrm{~cd}$ & $4.70 \mathrm{~cd}$ & $4.70 \mathrm{cdef}$ & $3.53 \mathrm{~h}$ \\
\hline
\end{tabular}

Means followed by the same letters within each column do not differ significantly according to Duncan's Multiple Range test at the $5 \%$ level. 
Table (6): Path coefficients (direct and joint effects) of snap pod yield and its related traits in bean for fall season 2011.

\begin{tabular}{|c|c|c|c|c|c|c|c|c|c|c|c|}
\hline Characters & $\mathrm{X1}$ & $\mathbf{X} 2$ & $\mathbf{X 3}$ & $\mathrm{X} 4$ & X5 & X6 & $\mathbf{X} 7$ & $\mathrm{X8}$ & X9 & $\mathbf{X 1 0}$ & $\mathbf{r}_{\mathrm{xy}}$ \\
\hline Plant height $\mathrm{X1}$ & $\underline{0.1911}$ & -0.0052 & 0.0350 & -0.0001 & 0.0274 & 0.0041 & 0.0676 & 0.0617 & \begin{tabular}{|l|}
-0.0297 \\
\end{tabular} & 0.0591 & 0.411 \\
\hline Number of branches/plant X2 & -0.0052 & $\underline{0.1929}$ & 0.0287 & -0.0025 & 0.0657 & -0.0123 & 0.0969 & 0.0778 & -0.0968 & 0.0717 & 0.417 \\
\hline Pod length $\mathrm{X3}$ & $\overline{0.0780}$ & 0.0646 & $\underline{\mathbf{0 . 0 8 5 8}}$ & -0.0021 & 0.1010 & 0.0011 & 0.0953 & 0.0872 & -0.0824 & 0.0715 & 0.500 \\
\hline Pod width $X 4$ & 0.0040 & 0.0939 & 0.0353 & -0.0052 & 0.0830 & -0.0023 & 0.0532 & 0.0345 & \begin{tabular}{|l|}
-0.0884 \\
\end{tabular} & 0.0579 & 0.266 \\
\hline Pod thickness X5 & -0.0227 & -0.0550 & -0.0376 & 0.0019 & -0.2306 & 0.0007 & -0.074 & -0.1253 & 0.0439 & -0.1176 & $\begin{array}{l}-0.617 \\
\end{array}$ \\
\hline Number of seeds/pod X6 & 0.0172 & -0.0521 & 0.0021 & 0.0003 & -0.0035 & $\underline{0.0457}$ & 0.0497 & 0.0636 & 0.0577 & -0.0067 & 0.174 \\
\hline Pod weight $\mathrm{X} 7$ & 0.0556 & 0.0804 & 0.0352 & -0.0012 & 0.0740 & 0.0098 & $\underline{0.2324}$ & 0.1317 & -0.0379 & 0.1059 & 0.686 \\
\hline Number of pods/plant X8 & 0.0424 & 0.0540 & 0.0269 & -0.0006 & 0.1040 & 0.0105 & 0.1102 & $\underline{0.2779}$ & -0.0343 & 0.1331 & 0.724 \\
\hline 100- seeds weight $\mathrm{X9}$ & 0.0285 & 0.0935 & 0.0354 & -0.0023 & 0.0507 & -0.0132 & 0.0442 & 0.0478 & -0.1995 & 0.0379 & 0.123 \\
\hline Early yield X10 & 0.0590 & 0.0723 & 0.0321 & -0.0016 & 0.1418 & -0.0016 & 0.1288 & 0.1934 & \begin{tabular}{|c|}
-0.0395 \\
\end{tabular} & $\underline{0.1912}$ & 0.776 \\
\hline
\end{tabular}

characters represented the casual variables. The matrixes of direct and joint effects for the ten yield-related traits on pod yield are shown in Table (6). The direct contribution of the number of pods per plant was the highest value $(\mathrm{p}=0.2779)$ followed by pod weight, the number of branches per plant, early yield, plant height, pod length and the number of seeds per pod, whereas pod thickness had a maximum negative direct effect on pod yield $(\mathrm{p}=-0.2306)$ followed by 100 -seed weight and pod width. From the results of this season, it could be concluded that the number of pods/plant, pod weight, pod thickness, 100-seed weight, the number of branches/plant, early yield and plant height were the most important contributing characters towards pod yield of bean. The number of branches/plant is considered an important trait to the pod yield followed by pod thickness, pod weight, plant height, the number of pods /plant and pod length. Their indirect effects contributed 13.50, 12.92, 9.91, 8.57, 7.20 and 5.85\% (Table 7). From the previous results of path analysis of the first season, it could be concluded that the number of branches/plant, plant height, the number of pods/plant and pod weight were the most important contributing characters to the total variability of the tested beans.

The early yield recorded the highest value in the second season for its direct contribution $(p=0.6376)$ followed by the number of pods per plant, the number of seeds per pod and pod length. On the other hand, the direct effects of 100 -seed weight, pod thickness, pod weight and plant height were positive and of secondary importance recording $(\mathrm{p}=0.0191),(\mathrm{p}=0.0844)$, $(\mathrm{p}=0.0731)$ and $(\mathrm{p}=0.0654)$, respectively. Whereas, pod width and the number of branches/plant were negative and had insignificant effects (Table 8). Early yield recorded the highly relative important direct effect $27.946 \%$ followed by the number of pods/plant, the number of seeds per pod, pod width and pod length. Results showed clearly that the relative important indirect effect of the number of pods/plant $13.11 \%$ and pod length $12.02 \%$ were the important traits causing variation followed by pod width, pod weight, plant height, pod thickness and the number of branches/plant (Table 9). 
Table (7): Direct and indirect effects of yield components and their relative importance in snap pod yield of bean for fall season 2011.

\begin{tabular}{|c|c|c|c|c|c|c|c|c|c|}
\hline \multirow[t]{2}{*}{ Characters } & \multicolumn{3}{|c|}{ Direct effect $X_{i}$} & \multicolumn{3}{|c|}{$\begin{array}{c}\text { Indirect effect } \mathrm{X}_{\mathrm{i}} / \\
\text { X's }\end{array}$} & \multicolumn{3}{|c|}{ Total effect } \\
\hline & Effects & CD* & RI \% & Effects & CD* & RI \% & Effects & CD* & RI \% \\
\hline Plant height $\mathrm{X1}$ & 0.1911 & 0.0365 & 2.8247 & 0.2199 & 0.0840 & 8.5741 & 0.4110 & 0.1206 & 11.3988 \\
\hline Number of ranches/plant X2 & 0.1929 & 0.0372 & 2.8781 & 0.2293 & 0.0884 & 13.5048 & 0.4222 & 0.1256 & 16.3829 \\
\hline Pod length $\mathrm{X} 3$ & 0.0858 & 0.0074 & 0.5691 & 0.2717 & 0.0466 & 5.8486 & 0.3574 & 0.0540 & 6.4177 \\
\hline Pod width X4 & -0.0052 & 0.0000 & 0.0021 & $\mathbf{0 . 1 3 8 0}$ & -0.0014 & 0.2563 & 0.1328 & -0.0014 & 0.2584 \\
\hline Pod thickness X5 & -0.2306 & 0.0532 & 4.1155 & -0.2730 & 0.1259 & 12.9223 & -0.5036 & 0.1791 & $\mathbf{1 7 . 0 3 7 8}$ \\
\hline Number of seeds/pod X6 & 0.0457 & 0.0021 & 0.1615 & 0.1643 & 0.0150 & 1.2564 & 0.2100 & 0.0171 & 1.4179 \\
\hline Pod weight $\mathrm{X} 7$ & 0.2324 & 0.0540 & 4.1800 & 0.1997 & 0.0929 & 9.9101 & 0.4322 & 0.1469 & 14.0901 \\
\hline Number of pods/plant X8 & 0.2779 & 0.0772 & 5.9727 & 0.0988 & 0.0549 & 7.1969 & 0.3766 & 0.1321 & 13.1696 \\
\hline 100- seeds weight $\mathrm{X9}$ & -0.1995 & 0.0398 & 3.0789 & 0.0379 & -0.0151 & 1.1687 & -0.1616 & 0.0247 & 4.2476 \\
\hline Early yield $\mathrm{X} 10$ & 0.1912 & 0.0366 & 2.8288 & 0.0000 & 0.0000 & 0.0000 & 0.1912 & 0.0366 & 2.8288 \\
\hline Total D+I & & & & & & & 1.8682 & 0.8353 & 87.2496 \\
\hline Residual & & & & & & & & 0.1647 & 12.7504 \\
\hline Total & & & & & & & 1.8682 & 1.000 & 100.000 \\
\hline
\end{tabular}

$\mathrm{CD}^{*}=$ Coefficient of determination.

RI \% = Relative efficiency.

Table (8): Path coefficients (direct and joint effects) of snap pod yield and its related traits in bean for summer season 2012.

\begin{tabular}{|c|c|c|c|c|c|c|c|c|c|c|c|}
\hline Characters & $\mathbf{X 1}$ & $\mathbf{X} 2$ & $\mathbf{X} 3$ & $\mathrm{X} 4$ & X5 & X6 & $\mathbf{X} 7$ & X8 & X9 & X10 & $\mathbf{r x y}$ \\
\hline Plant height X1 & 0.0654 & -0.0082 & 0.0406 & -0.0613 & -0.0271 & 0.0131 & 0.0264 & 0.0445 & 0.0041 & 0.2474 & 0.3450 \\
\hline $\begin{array}{l}\text { Number of branches/plant } \\
\text { X2 }\end{array}$ & 0.0163 & -0.0329 & 0.0460 & -0.0271 & -0.0175 & $-\mathbf{0 . 0 3 1 2}$ & 0.0018 & 0.0664 & 0.0059 & 0.2264 & 0.2540 \\
\hline Pod length X3 & 0.0172 & -0.0098 & 0.1545 & -0.0801 & -0.0421 & $-\mathbf{0 . 0 4 3 2}$ & 0.0195 & 0.0708 & 0.0061 & 0.3041 & 0.3970 \\
\hline Pod width X4 & 0.0253 & -0.0056 & 0.0782 & -0.1583 & -0.0226 & 0.0060 & 0.0123 & 0.0080 & 0.0070 & 0.2327 & 0.1830 \\
\hline Pod thickness X5 & -0.0210 & 0.0068 & -0.0771 & 0.0424 & 0.0844 & 0.0532 & -0.0092 & -0.0517 & -0.0019 & -0.2270 & -0.2010 \\
\hline Number of seeds/pod X6 & 0.0047 & 0.0057 & -0.0368 & -0.0052 & 0.0247 & 0.1816 & 0.0085 & 0.0010 & -0.0027 & -0.0185 & 0.1630 \\
\hline Pod weight $\mathrm{X7}$ & 0.0237 & -0.0008 & 0.0413 & -0.0268 & -0.0106 & 0.0212 & 0.0731 & 0.1097 & 0.0057 & 0.3316 & 0.5680 \\
\hline Number of pods/plant $\mathrm{X8}$ & 0.0146 & -0.0110 & 0.0549 & -0.0063 & -0.0219 & 0.0009 & 0.0402 & 0.1994 & 0.0063 & 0.4718 & 0.7490 \\
\hline 100- seeds weight $\mathrm{X9}$ & 0.0142 & -0.0102 & 0.0493 & -0.0584 & -0.0084 & -0.0260 & 0.0218 & 0.0664 & 0.0191 & 0.1843 & 0.2520 \\
\hline Early yield X10 & 0.0254 & -0.0117 & $\mathbf{0 . 0 7 3 7}$ & -0.0578 & $-\mathbf{0 . 0 3 0 0}$ & -0.0053 & $\mathbf{0 . 0 3 8 0}$ & 0.1476 & 0.0055 & 0.6376 & $\mathbf{0 . 8 2 3 0}$ \\
\hline
\end{tabular}


Table (9): Direct and indirect effects of yield components and their relative importance in snap pod yield of bean for summer season 2012.

\begin{tabular}{|c|c|c|c|c|c|c|c|c|c|}
\hline \multirow[t]{2}{*}{ Characters } & \multicolumn{3}{|c|}{$\begin{array}{c}\text { Direct effect } \\
\mathbf{X i}\end{array}$} & \multicolumn{3}{|c|}{$\begin{array}{c}\text { Indirect effect } \\
\text { Xi / X's }\end{array}$} & \multicolumn{3}{|c|}{ Total effect } \\
\hline & Effects & CD* & RI \% & Effects & CD* & RI \% & Effects & CD* & RI \% \\
\hline $\begin{array}{ll}\text { Plant height } & \text { X1 }\end{array}$ & 0.0654 & 0.0043 & 0.2939 & 0.2796 & 0.0366 & 4.2493 & 0.3450 & 0.0408 & 4.5432 \\
\hline Number of branch/plantX2 & -0.0329 & 0.0011 & 0.0745 & 0.2706 & -0.0178 & 1.9115 & 0.2377 & -0.0167 & 1.9860 \\
\hline Pod length X3 & 0.1545 & 0.0239 & 1.6410 & 0.2351 & 0.0727 & 12.0216 & 0.3896 & 0.0965 & 13.6626 \\
\hline $\begin{array}{ll}\text { Pod width } & \text { X4 }\end{array}$ & -0.1583 & 0.0251 & 1.7231 & 0.2435 & -0.0771 & 6.2837 & 0.0851 & -0.0520 & 8.0067 \\
\hline Pod thickness $\mathrm{X5}$ & 0.0844 & 0.0071 & 0.4893 & -0.2366 & $-\mathbf{0 . 0 3 9 9}$ & 3.9778 & -0.1522 & -0.0328 & 4.4670 \\
\hline Number of dry seeds /podX6 & 0.1816 & $\mathbf{0 . 0 3 3 0}$ & 2.2663 & -0.0117 & -0.0042 & 0.7679 & 0.1699 & 0.0287 & 3.0342 \\
\hline $\begin{array}{ll}\text { Pod weight } & \text { X7 }\end{array}$ & 0.0731 & 0.0053 & 0.3668 & 0.4470 & 0.0653 & 4.4887 & 0.5200 & 0.0706 & 4.8556 \\
\hline Number of pods/plant $\quad$ X8 & 0.1994 & 0.0398 & 2.7340 & 0.4782 & 0.1907 & 13.1106 & 0.6776 & 0.2305 & 15.8446 \\
\hline 100- seeds weight $\quad$ X9 & 0.0191 & 0.0004 & 0.0250 & 0.1843 & 0.0070 & 0.4828 & 0.2033 & 0.0074 & $\mathbf{0 . 5 0 7 7}$ \\
\hline Early yield $\quad$ X10 & 0.6376 & 0.4066 & 27.9460 & 0.0000 & 0.0000 & 0.0000 & 0.6376 & 0.4066 & 27.9460 \\
\hline Total D+I & & & & & & & 3.1138 & 0.7796 & 84.8536 \\
\hline Residual & & & & & & & & 0.2204 & 15.1464 \\
\hline Total & & & & & & & 3.1138 & 1.00 & $\mathbf{1 0 0 . 0 0}$ \\
\hline
\end{tabular}

Results in Table (10) of path coefficient analysis of the third season showed that early yield had the highest and positive effect on pod yield followed by pod weight, pod width and plant height.The maximum amount of negative direct effect was related to direct effect of pod thickness $(-0.1396)$ the next trait was100-seeds weight and the number of branches/plant followed by the number of pods/plant, pod length and the number of seeds/pod. From results in Table (11) showed that the early yield was the maximum relative important trait under direct effect to the total yield variability followed by pod weight, and pod thickness. Pod weight recorded highly relative important indirect effect to the total yield variation followed by pod thickness, pod width, the number of pods/plant, the number of branches/plant, 100seeds weight and pod length as the second important contributing characters towards pod yield of bean plants in the third season. Therefore, the relative importance of these characters to the total yield variability were, $12.65,10.40,5.35$, $3.65,3.33,2.59$ and $1.39 \%$, respectively.

In the fourth season, early yield recorded the highest positive and direct effect followed by pod weight, pod thickness, pod length, the number of seeds per pod, 100-seeds weight and pod width. On the other hand the number of pods/plan had an important negative direct and not significant effects and the next traits were plant height and the number of branches/plant (Table12). The highest value of relative important direct effect was given by early yield followed by the number of pods/plant, pod weight, pod thickness and pod length (Table13).The results showed clearly that the relative important indirect effect of the number of pods/plant, pod length, pod weight and pod thickness were the important traits to yield variation. 
Table (10): Path coefficients (direct and joint effects) of snap pod yield and its related traits in bean for fall season 2012.

\begin{tabular}{|c|c|c|c|c|c|c|c|c|c|c|c|}
\hline Characters & X1 & $\mathrm{X} 2$ & X3 & X4 & X5 & X6 & $\mathrm{X} 7$ & X8 & $\mathbf{X 9}$ & $\mathrm{X10}$ & $\mathbf{r x y}$ \\
\hline Plant height X1 & 0.0240 & -0.0064 & -0.0009 & 0.0042 & -0.0017 & -0.0002 & 0.0136 & -0.0078 & -0.0285 & 0.0499 & 0.046 \\
\hline $\begin{array}{l}\text { Number of } \\
\text { branch/plant X2 }\end{array}$ & 0.0031 & -0.0494 & -0.0032 & 0.0238 & 0.0346 & 0.0000 & 0.0297 & $-0.0201 \mid$ & -0.0419 & 0.3133 & 0.29 \\
\hline pod length $\mathrm{X} 3$ & 0.0008 & -0.0060 & -0.0268 & 0.0359 & 0.0647 & 0.0000 & 0.0821 & -0.0155 & -0.0204 & 0.2803 & 0.395 \\
\hline pod width $\mathrm{X} 4$ & 0.0009 & -0.0111 & -0.0090 & 0.1064 & 0.0468 & 0.0001 & 0.0583 & -0.0128 & -0.0504 & 0.1799 & 0.309 \\
\hline pod thickness X5 & 0.0003 & 0.0123 & 0.0124 & -0.0356 & -0.1396 & $-\mathbf{0 . 0 0 2 0}$ & -0.0663 & 0.0244 & 0.0123 & -0.4110 & -0.593 \\
\hline $\begin{array}{l}\text { Number of seeds / } \\
\text { podX6 }\end{array}$ & 0.0005 & 0.0001 & -0.0001 & -0.0005 & -0.0235 & -0.0118 & 0.0483 & -0.0053 & -0.0113 & 0.0216 & 0.018 \\
\hline Pod weight $\mathrm{X} 7$ & 0.0016 & -0.0073 & -0.0109 & 0.0306 & 0.0458 & -0.0028 & 0.2023 & $-0.0257 \mid$ & -0.0142 & 0.3935 & 0.613 \\
\hline $\begin{array}{l}\text { Number of } \\
\text { pods/plant X8 }\end{array}$ & 0.0041 & -0.0214 & -0.0090 & 0.0295 & 0.0736 & -0.0014 & 0.1121 & -0.0463 & -0.0197 & 0.5256 & 0.647 \\
\hline $\begin{array}{l}\text { 100- seeds weight } \\
\text { X9 }\end{array}$ & 0.0065 & -0.0196 & -0.0052 & 0.0508 & 0.0162 & -0.0013 & 0.0271 & -0.0086 & -0.1057 & 0.1698 & 0.13 \\
\hline Early yield X10 & 0.0018 & $-\mathbf{0 . 0 2 3 0}$ & -0.0111 & 0.0284 & 0.0852 & -0.0004 & 0.1181 & $|-0.0361|$ & -0.0266 & 0.6738 & 0.81 \\
\hline
\end{tabular}

Table (11): Direct and indirect effects of yield components and their relative importance in snap pod yield of bean for fall season 2012 .

\begin{tabular}{|c|c|c|c|c|c|c|c|c|c|}
\hline \multirow[b]{2}{*}{ Characters } & \multicolumn{3}{|c|}{ Direct effect $\mathrm{Xi}$} & \multicolumn{3}{|c|}{ Indirect effect Xi / X's } & \multicolumn{3}{|c|}{ Total effect } \\
\hline & Effects & CD* & RI \% & Effects & CD* & RI \% & Effects & CD* & RI \% \\
\hline \begin{tabular}{ll|} 
Plant height & X1 \\
\end{tabular} & 0.0240 & 0.0006 & 0.0416 & $\mathbf{0 . 0 2 2 0}$ & 0.0011 & 0.3923 & 0.0460 & 0.0016 & 0.4339 \\
\hline $\begin{array}{l}\text { Number of } \\
\text { branch/plant } \mathrm{X} 2\end{array}$ & -0.0494 & 0.0024 & 0.1762 & 0.3363 & -0.0332 & 3.3296 & 0.2869 & -0.0308 & 3.5058 \\
\hline Pod length $\mathrm{X3}$ & -0.0268 & 0.0007 & 0.0518 & 0.4270 & -0.0229 & 1.9299 & 0.4002 & -0.0222 & 1.9817 \\
\hline $\begin{array}{ll}\text { Pod width } & \text { X4 } \\
\end{array}$ & 0.1064 & 0.0113 & 0.8173 & 0.2218 & 0.0472 & 5.3490 & $\mathbf{0 . 3 2 8 2}$ & $\mathbf{0 . 0 5 8 5}$ & 6.1662 \\
\hline Pod thickness X5 & -0.1396 & 0.0195 & 1.4074 & \begin{tabular}{|l|}
-0.4427 \\
\end{tabular} & 0.1236 & 10.4016 & -0.5823 & 0.1431 & 11.8089 \\
\hline $\begin{array}{l}\text { Number of dry } \\
\text { seeds/pod X6 }\end{array}$ & -0.0118 & 0.0001 & 0.0101 & 0.0533 & -0.0013 & 0.1480 & 0.0414 & -0.0011 & 0.1581 \\
\hline $\begin{array}{ll}\text { Pod weight } & \text { X7 } \\
\end{array}$ & 0.2023 & 0.0409 & 2.9526 & $\mathbf{0 . 3 5 3 7}$ & 0.1431 & 12.6516 & 0.5559 & 0.1840 & 15.6041 \\
\hline $\begin{array}{l}\text { Number of pods/plant } \\
\text { X8 }\end{array}$ & -0.0463 & 0.0021 & 0.1550 & 0.5059 & -0.0469 & 3.6475 & 0.4596 & -0.0447 & 3.8025 \\
\hline $\begin{array}{l}\text { 100-dry seeds weight } \\
\text { X9 }\end{array}$ & $-0.1057 \mid$ & 0.0112 & 0.8058 & 0.1698 & -0.0359 & 2.5898 & 0.0641 & -0.0247 & 3.3956 \\
\hline Early yield X10 & 0.6738 & 0.4540 & 32.7676 & \begin{tabular}{|l|}
0.0000 \\
\end{tabular} & 0.0000 & 0.0000 & 0.6738 & 0.4540 & 32.7676 \\
\hline Total D+I & & & & & & & 2.2737 & 0.7147 & 79.6246 \\
\hline Residual & & & & & & & & 0.2823 & 20.3754 \\
\hline Total & & & & & & & 2.2737 & 1.000 & 100.000 \\
\hline
\end{tabular}


Table (12): Path coefficients (direct and joint effects) of snap pod yield and its related traits in bean for summer season 2013.

\begin{tabular}{|c|c|c|c|c|c|c|c|c|c|c|c|}
\hline Characters & X1 & $\mathbf{X} 2$ & $\mathbf{X 3}$ & X4 & X5 & X6 & $\mathbf{X 7}$ & $\mathbf{X 8}$ & X9 & $\mathbf{X 1 0}$ & $\mathbf{r x y}$ \\
\hline Plant height X1 & -0.0639 & -0.0081 & 0.0608 & 0.0033 & -0.0136 & 0.0062 & 0.0110 & -0.0492 & 0.0040 & 0.2935 & 0.244 \\
\hline $\begin{array}{l}\text { Number of } \\
\text { branches/plant X2 }\end{array}$ & -0.0105 & -0.0495 & 0.0882 & 0.0042 & -0.0913 & -0.0087 & 0.0112 & -0.0832 & 0.0108 & 0.3466 & 0.218 \\
\hline Pod length $\mathrm{X} 3$ & -0.0231 & -0.0260 & 0.1680 & 0.0070 & -0.1386 & -0.0016 & 0.0609 & -0.1063 & 0.0081 & $\mathbf{0 . 5 3 5 6}$ & 0.484 \\
\hline Pod width & -0.0142 & -0.0139 & $\mathbf{0 . 0 7 9 0}$ & 0.0149 & -0.0962 & 0.0078 & 0.0569 & -0.0608 & 0.0113 & 0.4293 & 0.414 \\
\hline Pod thickness X5 & 0.0047 & 0.0245 & -0.1264 & -0.0078 & 0.1844 & 0.0025 & $-\mathbf{0 . 0 3 7 3}$ & 0.0890 & -0.0086 & -0.5280 & -0.403 \\
\hline Number of seeds / podX6 & -0.0064 & 0.0069 & -0.0042 & 0.0019 & 0.0074 & 0.0621 & $\mathbf{0 . 0 3 1 8}$ & -0.0289 & -0.0040 & 0.0855 & 0.152 \\
\hline Pod weight X7 & -0.0032 & -0.0025 & 0.0464 & 0.0038 & -0.0312 & 0.0089 & 0.2205 & -0.1317 & 0.0013 & 0.4995 & 0.612 \\
\hline $\begin{array}{l}\text { Number of pods/plant } \\
\text { X8 }\end{array}$ & -0.0136 & -0.0178 & 0.0773 & 0.0039 & -0.0710 & 0.0078 & 0.1257 & -0.2311 & 0.0083 & 0.7655 & 0.655 \\
\hline 100- seeds weight $\quad$ X9 & -0.0077 & -0.0160 & 0.0405 & 0.0050 & -0.0474 & -0.0075 & 0.0088 & -0.0575 & $\mathbf{0 . 0 3 3 4}$ & 0.1633 & 0.115 \\
\hline Early yield X10 & -0.0197 & -0.0181 & 0.0948 & 0.0067 & -0.1025 & 0.0056 & 0.1160 & -0.1862 & 0.0058 & 0.9497 & 0.852 \\
\hline
\end{tabular}

Table (13): Direct and indirect effects of yield components and their relative importance in snap pod yield of bean for summer season 2013.

\begin{tabular}{|c|c|c|c|c|c|c|c|c|c|}
\hline \multirow{2}{*}{ Characters } & \multicolumn{3}{|c|}{ Direct effect $\mathrm{Xi}$} & \multicolumn{3}{|c|}{ Indirect effect Xi / X's } & \multicolumn{3}{|c|}{ Total effect } \\
\hline & Effects & CD* & RI \% & Effects & CD* & RI \% & Effects & CD* & RI \% \\
\hline & -0.0639 & 0.0041 & 0.1554 & 0.3079 & -0.0393 & 2.1888 & 0.2440 & -0.0353 & 2.3442 \\
\hline Number of branch/plant X2 & -0.0495 & 0.0025 & 0.0933 & 0.2780 & -0.0275 & 2.4286 & 0.2285 & -0.0251 & 2.5219 \\
\hline Pod length X3 & 0.1680 & 0.0282 & 1.0753 & 0.3651 & 0.1227 & 10.9817 & 0.5331 & 0.1509 & 12.0570 \\
\hline Pod width X4 & 0.0149 & 0.0002 & 0.0085 & 0.3482 & 0.0104 & 0.7523 & 0.3631 & 0.0106 & 0.7608 \\
\hline Pod thickness X5 & 0.1844 & 0.0340 & 1.2945 & -0.4824 & -0.1779 & 9.3430 & -0.2981 & -0.1439 & 10.6375 \\
\hline Number of seeds $\backslash$ pod X6 & 0.0621 & 0.0039 & 0.1470 & 0.0843 & 0.0105 & 0.7104 & 0.1464 & 0.0143 & 0.8574 \\
\hline Pod weight $\mathrm{X7}$ & 0.2205 & 0.0486 & 1.8521 & 0.3692 & 0.1628 & 10.6253 & 0.5897 & 0.2115 & 12.4774 \\
\hline Number of pods/plant X8 & -0.2311 & 0.0534 & 2.0331 & 0.7738 & -0.3576 & 13.6172 & 0.5427 & -0.3042 & 15.6502 \\
\hline 100-seeds weight X9 & 0.0334 & 0.0011 & 0.0426 & 0.1633 & 0.0109 & 0.4159 & 0.1968 & 0.0120 & 0.4584 \\
\hline Early yield X10 & 0.9497 & 0.9019 & 34.3473 & 0.0000 & 0.0000 & 0.0000 & 0.9497 & 0.9019 & 34.3472 \\
\hline Total D+I & & & & & & & 3.4960 & 0.7929 & 92.1121 \\
\hline Residual & & & & & & & & 0.2071 & 7.8879 \\
\hline Total & & & & & & & 3.4960 & 1.000 & 100.00 \\
\hline
\end{tabular}


The results of path analysis in the four seasons showed that early yield, pod weight, the number of pods per plant and the number of branches per plant were the most important contributing traits to the total yield variability of snap bean. It is concluded that these traits could be important for designing selection criteria index in snap bean breeding. Results of this experiment in the four seasons are in agreement with those obtained by Mohamed (1997) who found that the number of primary branches, the number of pods/plant and early yield had significant positive correlation with total snap pods yield. Path analysis revealed that the number of primary branches had the greatest direct effects on pod yield. Goncalves et al. (2003) found that yield per plant and the number of pods per plant were important variables included in the study that presented the best combinations of path coefficient and correlation, both positive and of high magnitude and both were superior to that of the variable the number of seeds per pod. Roy et al. (2006) found that pods/plant, 100-seeds weight, seeds/pod, plant height and pod length had positive direct effect on yield. Atilla (2007) found that pod weight, pod length and pod number per plant had the highest effects on yield. Salehi et al. (2008) found that there were positive and significant correlations between the number of seeds per pod, the number of pods per plant and pod length, with grain yield. Rai et al. (2010) reported that the number of pods/plant and the number of seeds/pod showed maximum direct effect on yield. Salehi et al. (2010) reported that path analysis showed that the maximum direct and positive effects were given by the number of seeds per pod. The only direct and negative effect was related to pod length. Krasu and Oz (2011) concluded that seeds yield/plant had the highest direct effect on 100- seeds weight and plant height. Sofi et al. (2011) observed that seeds yield was significantly associated with the number of pods/plant followed by 100 -seeds weight, seeds/pod and plant height. Mehra and Singh (2012) found that path coefficient analysis revealed that pods yield per plant and the number of pods per cluster were the most important traits affecting pods yield. Araujo et al. (2012) found that indirect selection for pods yield could be by using the number of pods per plant as a reference and, for indirect selection for pod yield, the characters average length of the pod, 100- seeds weight and pods yield considering the latter primary character for pod yield. Kulaza and Ciftci (2012) found positively significant relationships among yield and yield per plant, the number of branches per plant, the number of pods per plant. There were strong direct effects of the 1000-seeds weight, yield per plant and plant height on yield. Ahmed and Kamaluddin (2013) found that the number of seeds/pod, plant height and the number of pods/plant showed positive and significant association with yield. The number of pods/plant, 100 seeds weight and pod length had maximum positive direct effect on yield.

\section{REFERENCES}

Ahmed S. and Kamaluddin (2013). Correlation and path analysis for agro-morphological traits in rajmash beans under BaramullaKashmir region. African J. Agric. Res. 8(18): 2027-2032.

Araujo L.C., Gravina G.A., Marinho C.D., Almeida S.N.C., Daher R.F., and Junior A.T.A. (2012).Contribution of components of production on snap bean yield. Crop Breed. Applied Biotech. 12: 206-210.

Atilla, D. (2007).Variability, heritability and correlation studies in bean (Phaseolus vulgaris L.) genotypes. World J. Agric. Sci. 3(1): 12-16.

Board J.E., Kang M.S. and Harville B.G. (1997). Path analyses identify indirect selection criteria for yield of late planted soybean. Crop Sci. 37(3): 879-884.

Coimbra J.L.M., Guidolin A.F., Carvalho F.I.F. and Duarte I.A. (1998).Quantitative analysis of genetic and phenotypic parameters in bean (Phaseolus vulgaris L.). Pesquisa Agropecuaria Gaucha. 4: 157-165.

Coimbra J.L.M., Hemp S., Carvalho F.I.F. and Selva S.A. (1999). Adaptability and phenotypic stability of coloured bean (Phaseolus vulgaris L.) genotype in three distinct environments. Ciencia Rural. 29: 441-448. 
Dahiya A. Sharma S.K., Singh K.P. and Kumar A. (2000).Variability studies in French bean (Phaseolus vulgaris L.). Annals of Biology. 16: 201-204. (c.f. CAB Abst. 2000/2002).

Dewey D.R. and Lu K.H. (1959).A correlation and path coefficient analysis of components of crested wheat grass seed production. Agron. J. 51: 515-518.

Duncan D.B. (1955).Multiple range and multiple F test. Biometrics, 11: 1-42.

El-Sayed S.F. (1990). Comparative study on some common bean cultivars.1.Growth and yield components. J. Agric. Res., Tanta Univ. Egypt 16: $100-110$.

El-Sayed S. F. (1996). A comparative study on some snap bean genotypes under low temperature conditions. J. Agric. Res., Tanta Univ. Egypt 22: 191-203.

Escribano M.R., Ron A.M. and Amurrio J.M.(1994). Diversity in agronomical traits in common bean population from North Western Spain. Euphytica 76: 1-6.

Galal R.M. (2004). Genetic Studies for Improving Productivity of Beans (Phaseolus vulgaris L.). Ph.D. Thesis, Minia Univ. Egypt pp.191.

Goncalves M. C., Corea A. M., Destro D., Souza L. D., Alves S.T. and Souza L.C.F. (2003). Correlations and path analysis of common bean grain yield and its primary components. Crop Breeding and Applied Biotecnology. 3(3): 217-222.

Gravois K.A. and Helmes R.S. (1992). Path analysis of rice yield components as affected by seeding rate. Agrono. J. 84: 1- 4.

Krasu A. and OZ M. (2011). A study on coefficient analysis and association between agronomical characters in dry bean (Phaseolus vulgaris L.). Bulgarian J. Agric. Res. 16 (2):203-211.

Kulaza H. and Ciftci V. (2012). Relationships among yield components and selection criteria for seed yield improvement in bush bean (Phaseolus vulgaris L). Tarım Bilimleri Dergisi - J. Agri. Sci. 18: 257-262.

Kurek A. J., Carvalho F.I.F., Assmann I.C., Marchioro V.S. and Cruz P.J. (2001). Path analysis for indirect seletion of bean field. Rev. Brasil. Agro. 7: 29-32.
Mehra D. and Singh D. K. (2012). Path coefficient analysis for pod yield in French bean. Veg. Sci. 39 (2): 192-194.

Mohamed M.F. (1997). Screening of some common bean (Phaseolus vulgaris L.) cultivars for production in Southern Egypt and path coefficient analysis for green pod yield. Assiut J. Agric. Sci. 28: 91-106.

Mohamed N.A. (2004). A Genetic Study on Common Bean (Phaseolus vulgaris L.). M.Sc. Thesis, Fac. Agric. Minia Univ. Egypt pp.115.

Nassar H.H. (1986). The relationship between yield and growth character in snap bean varieties. Ann. Agric. Sci., Fac. Agric., Ain Shams Univ., Egypt. 31: 1351-1360.

Rai N., Singh P.K., Verma A., Yadav P.K. and Choubey T. (2010). Hierarchical analysis for genetic variability in pole type French bean. Indian J. Hort., 67:150-153.

Ribeiro N. D., Junior L. H., Stroschein M. R. D. and Possebon S. B. (2003). Genotype x environment interaction in common bean yield and yield components. Crop Breeding and Applied Biotechnology, 3(1): 27-34.

Roy S.K., Abdul Karim M., Aminul A.K.M., Bari M.N., Main M.A.K. and Tetsushi H. (2006). Relationship between yield and its component characters of bush bean (Phaseolus vulgaris L.). South pacific studies, 27(1): 13-22.

Salehi M., Faramarzi A. and Mohebalipour N. (2010). Evaluation of different effective traits on seed yield of common bean (Phaseolus vulgaris L.) with path analysis. American-Eurasian J. Agric. and Environ. Sci. 9 (1): 52-54.

Salehi M., Tajik M. and Ebadi A.G. (2008). The study of relationship between different traits in common bean (Phaseolus vulgaris L.) with multivariate statistical methods. American-Eurasian J. Agric.and Environ. Sci. 3 (6): 806-809.

Singh S.P. (1999). Common Bean: Improvement in the Twenty-First Century. Kluwer Academic Publishers, @London, pp: 2-7.

Singh D.N., Nandi A. and Tripathy P. (1994). Genetic variability and character association in French bean (Phaseolus vulgaris L.). 
Indian J. Agric. Sci., 64: 114-116. [ c.f. HORTCD 1989/1997].

Sofi P.A., Zargar M.Y., Debouck D. and Graner A. (2011). Evaluation of common bean (Phaseolus vulgaris L.) germplasm under temperate conditions of Kashmir Valley. J. Phytol. 3(8):47-52.

Wright S. (1921). Correlation and causation. J. Agric. Res. 20:557-585.
Zhiwei Q., Yang X.U., Liu H., Yan C., Teng B., Qin Z.W., Xu X.Y., Liu H.Y., Yan C.G., Teng B. and Z. Dewei (1995). Evaluation of quality characteristics of the fresh pods of the bean (Phaseolus vulgaris L.) in breeds in Heilongjiang Province. Acta Hort. No. 402: 200-205. [c.f. CAB Abst. 1996/1998].

$$
\begin{aligned}
& \text { معامل المرور كنموذج احصائي لإنتقاء بعض السلالات الجديدة من الفاصوليا } \\
& \text { رافت محمد جلال- وفاء وهبة محمد شافعى* ـ سحر عبدالعزيز فرج* } \\
& \text { قسم الخضر - معهد بحوث البساتين و * المعمل المركزى لبحوث التصميم و التحليل الاحصائى الزئ } \\
& \text { مركز البحوث الزر اعية ــ الجيزة - مصر ليحر }
\end{aligned}
$$

